\title{
PREPRINT
}

Note: This is a preprint of a manuscript being prepared for publication. Contents of this paper should not be quoted or referred to without permission of the author(s).

(submitted to Proceedings of 1995 MRS; Symposium A)

\section{New Insight into Damage-Related Phenomena in Si Implanted under Extreme Conditions}

\author{
O. W. Holland and J. D. Budai \\ Oak Ridge National Laboratory \\ Bldg. 3003 MS-6048 \\ Oak Ridge, Tennessee 37831-6048 \\ Bent Nielsen \\ Brookhaven National Laboratory \\ Upton, New York 11973
}

\begin{abstract}
"The submitted manuscript has been authored by a contractor of the U.S. Government under contract number DE-AC05-96OR22464. Accordingly, the U.S. Government retains a nonexclusive, royalty-free license to publish or reproduce the published form of this contribution, or allow others to do so, for U.S. Government purposes."
\end{abstract}

\author{
Prepared by the \\ Oak Ridge National Laboratory \\ Oak Ridge, Tennessee 37831 \\ managed by \\ Lockheed Martin Energy Research Corp. \\ for the \\ U.S. DEPARTMENT OF ENERGY \\ under contract DE-AC05-96OR22464.
}

January 1996

PREPRINT 


\title{
NEW INSIGHT INTO DAMAGE-RELATED PHENOMENA
}

IN Si IMPLANTED UNDER EXTREME CONDITIONS

\author{
O. W. HOLLAND, BENT NIELSEN ${ }^{+}$, AND J. D. BUDAI
}

Oak Ridge National Laboratory, P. O. Box 2008, Oak Ridge, TN 37831-6048

+Brookhaven National Laboratory, Upton, NY 11973.

\section{ABSTRACT}

New insight into damage formation in $\mathrm{Si}(100)$ during self-ion irradiation is gained from processing under extreme conditions. Dislocations form in the near-surface as a result of lattice relaxation in response to strain produced by precursor defects which are shown to be vacancy-type by positron analysis. A model to account for these defects and their distribution is presented. A novel technique is demonstrated which utilizes a subsequent implantation as a depth specific probe to manipulate the vacancy-type defects. Aspects of damage growth which emerge from the probe results are discussed.

\section{INTRODUCTION}

Ion-induced damage growth in single-crystal $\mathrm{Si}$ at or near room temperature has been widely studied by a number of investigators $(1)$. While it is generally accepted that damage production decreases with increasing temperature, details of the formation mechanism(s) are still lacking for elevated temperature irradiations $\left(\geq 200^{\circ} \mathrm{C}\right)$. Below this temperature range, damage growth is interrupted at moderate fluences by a spontaneous crystalline-to-amorphous transition of the lattice ${ }^{(2)}$, while above the transition temperature, it can be investigated to arbitrarily large fluence. Hightemperature processing, therefore, greatly extends the boundary for exploring damagerelated phenomena into regimes where new effects may be anticipated. While such prospects are clearly exciting, a better understanding of ion-solid interactions under extreme conditions also serves to underpin evolving ion-beam technologies such as separation by implantation of oxygen (SIMOX); a high-temperature process for synthesis of a silicon-on-insulator (SOI) material by high-dose $\mathrm{O}^{+}$-implantation(3-8)

Mechanisms responsible for the growth of damage in single-crystal Si during selfion irradiation at high temperature are investigated. The use of self-ions in the present study allowed the basic mechanisms of damage formation to be investigated without interference from chemical effects associated with a reactive ion specie. The focus of this paper will be on damage accumulation within the superficial layer ahead of the ions' end-of-range (eor), where an accumulation of precursor defects is shown to result in dislocation formation. The precursor defects are shown to be of a vacancy type by positron annihilation spectrometry. A mechanism based solely on the kinematics of the ion-atom interaction (and not the chemistry) is proposed to account for the presence of these vacancy-type defects in the superficial layer. Finally, a technique involving selfion implantation in the proximity of these vacancy-type defects is shown to reduce their concentration yielding a more defect-free layer. Insight gained from these results into the nature of the ion-induced damage process is discussed. Cross-section transmission electron microscopy (XTEM) and Rutherford backscattering (RBS)/channeling 
spectrometry are used to profile the ion-induced damage. The RBS spectra were acquired using $2.3 \mathrm{MeV} \mathrm{He}^{++}$-ions and a detector positioned to intercept backscattered ions at $160^{\circ}$.

\section{RESULTS AND DISCUSSION}

XTEM micrographs are shown in Fig. 1 from p-type Si(100) crystals implanted at $450^{\circ} \mathrm{C}$ with different fluences of $1.25 \mathrm{MeV} \mathrm{Si}^{+}$-ions. The resistivity of the $\mathrm{Si}$ crystals was nominally $10 \Omega-\mathrm{cm}$, and the average ion current density during implantation was $\leq 1 \mu \mathrm{A} / \mathrm{cm}^{2}$. Damage formed near the eor is seen to consist of a band of dislocations centered at $\sim 1.5 \mu \mathrm{m}$ which broadens with fluence. Surprisingly, the $3 \times 10^{17} \mathrm{~cm}^{-2}$ implanted sample is seen in Fig. 1(a) to be free of visible defects to a depth of $\sim 1.0 \mu \mathrm{m}$. This occurs despite the fact that the average number of displacements per atom (dpa) within this region is calculated at this dose to be $\sim 40$ by computer simulation using $\operatorname{TRIM}(9)$. Clearly, Frenkel pair recombination dominates at $450^{\circ} \mathrm{C}$ over those pointdefect reactions which result in damage formation. A unique morphology is seen at the higher fluences in Figs. 1(b) and 1(c). A distinct band of dislocations is present within the superficial layer centered near $\sim 0.45 \mu \mathrm{m}$. The width of the band increases with fluence and eventually expands to intersect both the surface and the advancing eor damage. Similar results have been reported for $\mathrm{O}^{+}$-implantation under nearly identical conditions $(10,11)$, but the use of self-ions indicates that these effects are intrinsic to these extreme conditions (i.e., high temperature and dose) and are not dependent upon the ion-solid chemistry. In ref. (10), it was shown that these dislocations are formed as a result of yielding of the lattice in response to an accumulation of precursor defects. The similarity of the self-ion results suggests a common mechanism.

Si SURFACE

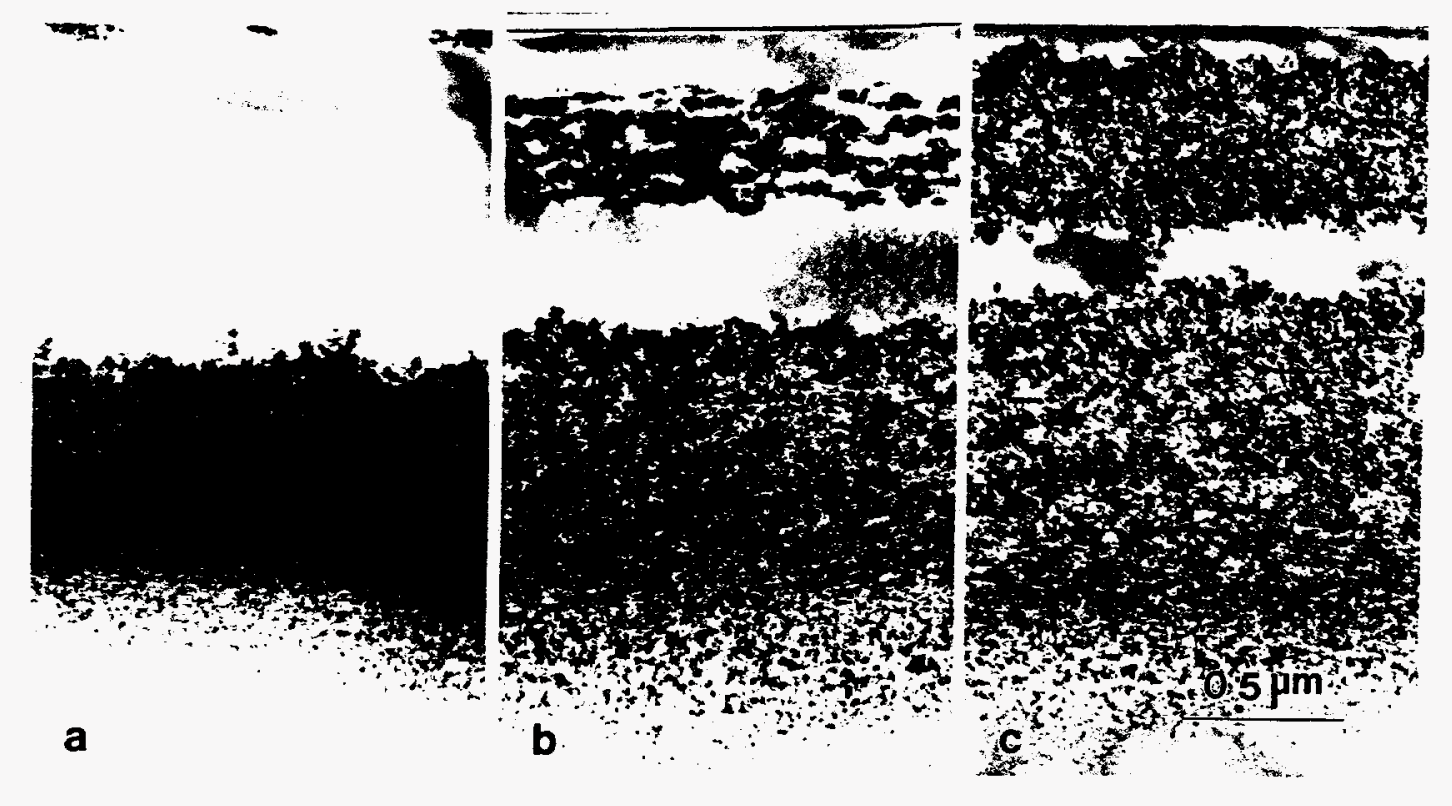

Fig. 1. XTEM micrographs from $\mathrm{Si}(100)$ crystals implanted at $450^{\circ} \mathrm{C}$ with $1.25 \mathrm{MeV}$ self-ions to a fluence of (a) $2 \times 10^{17} \mathrm{~cm}^{-2}$, (b) $3 \times 10^{17} \mathrm{~cm}^{-2}$, and (c) $5 \times 10^{17} \mathrm{~cm}^{-2}$. 
Identification of the precursor defects within the superficial layer prior to dislocation formation was done using positron annihilation techniques which are very sensitive to open volume defects in solids(12). The sensitivity of positrons to lattice defects is reflected in a quantity called the S-parameter which measures the Doppler broadening of the $511 \mathrm{keV}$ photon annihilation peak. The S-parameter is calculated by dividing the area of a fixed region in the center of the annihilation peak by the total peak area. Since a positron trapped within an open-volume defect interacts less frequently with the higher momentum, inner shell electrons, the width of the annihilation spectrum from such defects will be narrower (and the S-parameter larger) than from sites in undamaged crystal. The dependence of the S-parameter on the incident positron energy (depth) is shown in Fig. 2 for samples implanted with (a) $2 \times 10^{17}$ and (b) $5 \times 10^{17}$ $\mathrm{cm}^{-2}$; a dose range which spans the onset of dislocation growth within the superficial layer. At the lower dose, the S-parameter is seen to change rapidly over the ions' range with a peak value near $\sim 0.4 \mu \mathrm{m}$ much greater than $S_{b}=1$ for the bulk. This signifies the presence of open-volume defects within the superficial layer far in excess of the bulk concentration prior to dislocation formation. A much smaller S-parameter was measured in the sample implanted at the higher fluence of $5 \times 10^{17} \mathrm{~cm}^{-2}$. It is clear that dislocation formation during yielding reduces the concentration of vacancy-type defects within the superficial region.

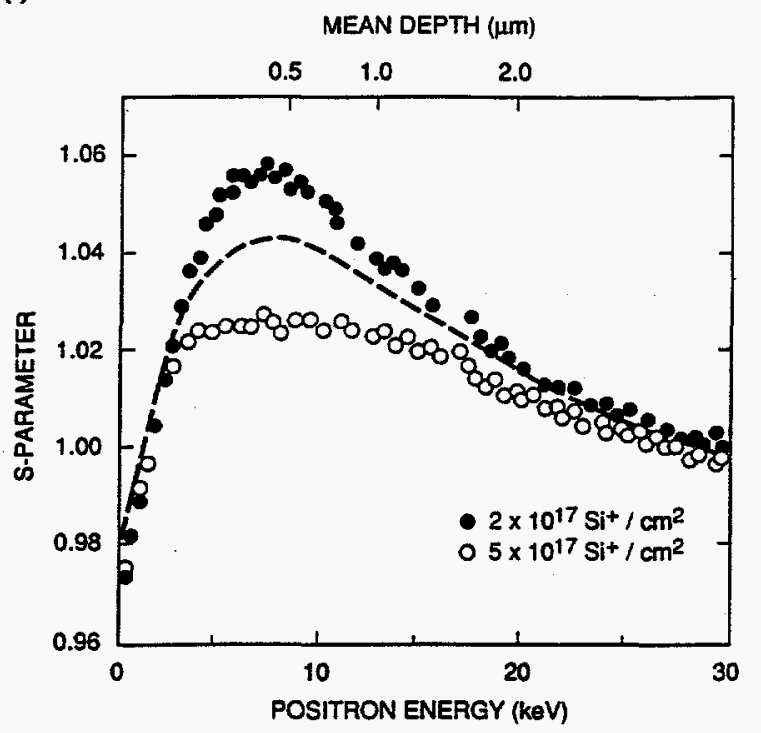

Fig. 2. S-parameter verses positron energy after implantation at $450^{\circ} \mathrm{C}$ with $1.25 \mathrm{MeV}$ self-ions to a dose of $2 \times 10^{17}$ and $5 \times 10^{17} \mathrm{~cm}^{-2}$. The dashed spectrum is from a sample dual implanted at $450^{\circ} \mathrm{C}$ with self-ions at $1.25 \mathrm{MeV}\left(2 \times 10^{17} \mathrm{~cm}^{-2}\right)$ and $300 \mathrm{keV}\left(2 \times 10^{15} \mathrm{~cm}^{-2}\right)$. The depth scale at the top of the figure corresponds to the average annihilation depth of the positrons.

The preponderance of vacancy-type defects within the superficial layer can be accounted for by a mechanism involving the spatial separation of Frenkel defects $(13,14)$. Momentum transfer to an interstitial, during Frenkel pair formation, will on average produce a spatial separation of the pair such that the interstitials are distributed to greater depths. TRIM calculations in Fig. 3 reveal that the vacancy excess produced at $1.25 \mathrm{MeV}$ by a self-ion fluence of $2 \times 10^{17} \mathrm{~cm}^{-2}$ persists over the top micron at a nominal value of 1 at. \% (followed by a much narrower distribution of excess interstitials). This 
closely matches the distribution of the vacancy-type defects within the sample, as well as yielding a reasonable value for their concentration. Thermal interdiffusion of defects may reduce the excess concentrations, especially near the boundary (between the excess vacancies and interstitials); but the excess distributions are preserved, in part, by interactions such as vacancy clustering which form less mobile defects.

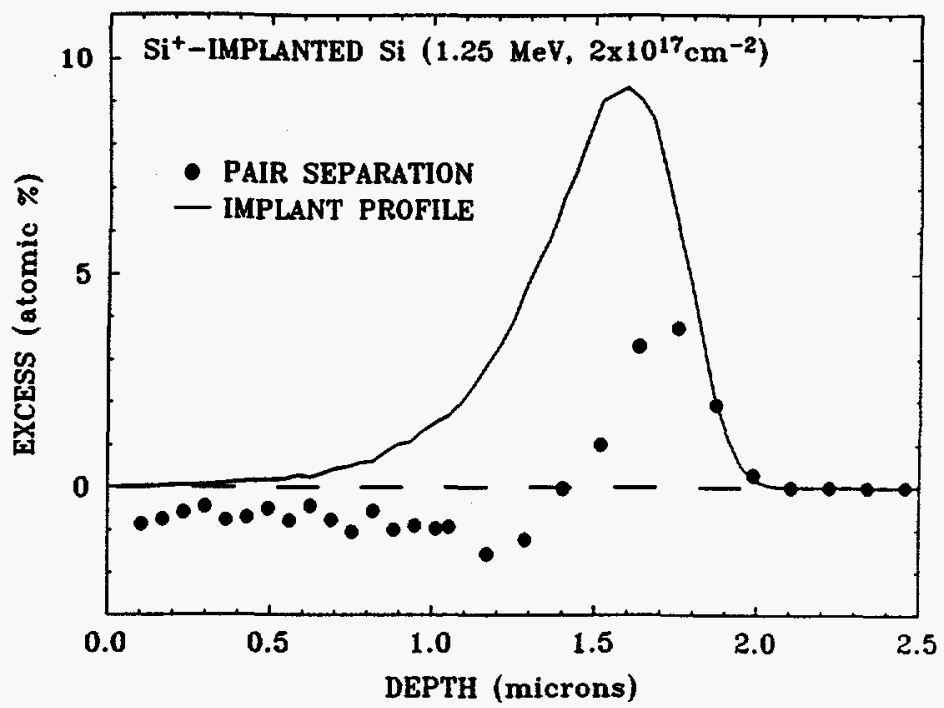

Fig. 3. TRIM calculations of $1.25 \mathrm{MeV}$ self-ion implantation in $\mathrm{Si}$ normalized to a fluence of $2 \times 10^{17} \mathrm{~cm}^{-2}$. The uncorrelated pairs, defined as the difference between the interstitial and vacancy distributions, is given by the solid circles. The distribution of the implanted self-ions is shown for reference.

While the focus to this point has clearly been upon ion-induced damage growth within the superficial layer, a more complete understanding of high-temperature effects must include some explanation for the damage behavior near the eor. Unlike the long incubation period within the superficial layer, dislocations form readily near the eor and, if anything, derive from an excess interstitial concentration. A strain-management technique developed to suppress dislocation formation during SIMOX processing(15), yielded insight into the nature of the damage process near the eor. This technique utilizes a self-ion beam as a spatially specific probe to manipulate pre-existing lattice defects. In particular, the beam was used to probe the vacancy-rich superficial layer formed by implanting $1.25 \mathrm{MeV}$ self-ions at $450^{\circ} \mathrm{C}$ to a fluence of $2 \times 10^{17} \mathrm{~cm}^{-2}$. The energy of the probe beam was set to $300 \mathrm{keV}$ so that the range of the ions $(\sim 0.4 \mu \mathrm{m})$ would match the depth of the maximum vacancy concentration. RBS spectra in Fig. 4 compare the aligned yield, before and after application of the probe, in a virgin lattice ( $\Delta$ and -, respectively); and in one previously implanted at $1.25 \mathrm{MeV}$ (• and +, respectively). In particular, attention should be focused within the superficial layer (over a depth of $\sim 0.5 \mu \mathrm{m}$ ) where any damage produced by this $300 \mathrm{keV}$ beam would be seen as an incremental change in the aligned yield. It is clear that the damage produced by the $300 \mathrm{keV}$ ions causes a marked increase in the aligned yield from the virgin crystal. This contrasts with the behavior in the $1.25 \mathrm{MeV}$ implanted sample where the aligned yield is unchanged indicating no additional accumulation of damage due to the probe irradiation. In fact, positron analysis in Fig. 2 (dashed curve) demonstrates that 
the $300 \mathrm{keV}$ beam actually reduced the concentration of vacancy-type defects formed by $1.25 \mathrm{MeV}$ self-ions. Presumably, this occurs as a result of an attraction between the atomic excess introduced by the beam and the vacancies resulting in mutial annihilation. (It is interesting to note the difference in sensitivity to vacancies between the two characterization techniques. While these defects are easily detected using positrons, the spectra in Fig. 4 show the RBS technique to be relatively insensitive.)

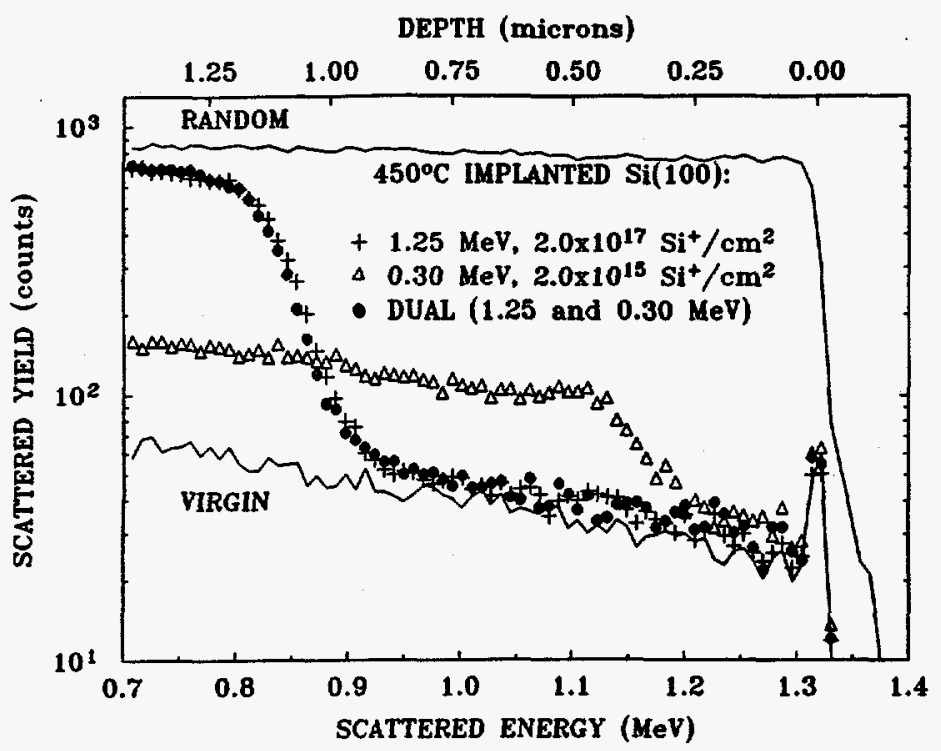

Fig. 4. Spectra comparing the [100] aligned yield from samples with and without a probe beam implantation at $450^{\circ} \mathrm{C}$. The probe consisted of a $2 \times 10^{15} \mathrm{~cm}^{-2}$ fluence of $300 \mathrm{keV}$ self-ions. The spectra show the effects of the probe in a virgin $\mathrm{Si}(100)$ crystal, and one predamaged at $1.25 \mathrm{MeV}$ with self-ions to a fluence of $2 \times 10^{17} \mathrm{~cm}^{-2}$. A random yield is also shown.

The probe results clearly show that the damage growth near the eor is affected by the state of the lattice, i.e., damage forms (under the conditions studied) within a virgin lattice but not in one with a high-vacancy concentration. The absence of damage near the probe's eor within the predamaged lattice suggests that cascade phenomena such as displacement spikes do not contribute to the damage process at elevated temperatures. A spike, first proposed by Brinkman $(16,17)$ to account for eor damage, is a violently disturbed region formed by the high density of atomic displacements produced by an ion as it slows down. Since the spike mechanism for forming damage involves the collective motion of a large number of atoms, a relative small density of pre-existing defects within the vicinity of a spike is not expected to significantly impact either its size or density. Therefore, the absence of eor damage within the predamaged lattice leaves one to conclude that displacement spikes are of no consequence at elevated temperatures and must be completely relaxed through defect recombination. Rather, it appears that eor damage forms in the virgin lattice as a result of the inability of the lattice to accommodate the atomic excess produced by implantation. Since a virgin lattice contains insufficient vacant sites to accommodate the implanted atoms, it is reasonable to assume that interstitial type defects will be formed. This is consistent with reports of interstitial-type defects near the eor such as the loops, rods, and intermediate defect configurations ${ }^{(18)}$. 


\section{CONCLUSIONS}

New effects related to ion-induced damage accumulation at high-temperature in $\mathrm{Si}(100)$ were demonstrated. The use of self-ions in this study showed the effects to be independent of the ion-solid chemistry. Mechanisms were elucidated, including one based on kinematic separation of Frenkel pairs to account for the vacancy excess within the superficial layer which gives rise to negative lattice strain. A technique utilizing a self-ion beam was shown to be effective in eliminating pre-existing, vacancy-type defects. The results from studying the interaction between the self-ions and pre-existing defects demonstrated that cascade effects are of little or no importance to damage accumulation at elevated temperatures, even near the eor. Rather, damage appears to form near the eor simply because implanted atoms cannot be accommodated within the lattice without damage formation.

\section{ACKNOWLEDGMENT}

This research was sponsored by Oak Ridge National Laboratory, managed by Lockheed Martin Energy Research Corp. for the U.S. Department of Energy under contract number DE-AC05-96OR22464, and Contract DE-AC02-76CH00016. D.S. Zhou is gratefully acknowledged for his efforts in providing XTEM results.

\section{REFERENCES}

1. See for example: James F. Gibbons, Proceedings of the IEEE 60, 1062 (1972); O. W. Holland, S. J. Pennycook, and Gerald L. Albert, Appl. Phys. Lett. 55, 2503 (1989); O. W. Holland and C. W. White, Nucl. Instr. Meth. B59, 353 (1991) and references within.

2. J. Linnros, R. G. Elliman, and W. L. Brown, J. Mater. Res. 3, 1208 (1988).

3. H. H. Hosack, T. W. Houston, and G. P. Pollack, Sol. State Tech. 12, 61 (1990).

4. M. A. Guerra, Sol. State Tech. 11, 75 (1990).

5. G. K. Celler and A. E. White, Mat. Res. Soc. Bull. 6, 40 (1992).

6. Laura Peters, "Semiconductor International," March 1993, p. 48.

7. O. W. Holland, T. P. Sjoreen, D. Fathy, and J. Narayan, Appl. Phys. Lett. 45, 10 (1984).

8. A. H. van Ommen and M. P. A. Viegers, Appl. Surf. Sci. 30, 383 (1987).

9. J. Biersack and L. Haggmark, Nucl. Instr. and Meth. 174, 257 (1980).

10. O. W. Holland, D. S. Zhou, and D. K. Thomas, Appl. Phys. Lett. 63, 896 (1993).

11. D. S. Zhou, O. W. Holland, and J. D. Budai, Appl. Phys. Lett. 63, 3580 (1993).

12. Peter J. Schultz and K. G. Lynn, Review of Modern Physics 60, 701 (1988).

13. A. M. Mazzone, Phys. Status Solids A 95, (1986) 149; and K. Touhouche, J. Jackman, and A. Yelon, Nucl. Instr. and Meth. B 80/81, (1993) 857.

14 O. W. Holland, Ling Xie, Bent Nielsen, and D. S. Zhou, J. of Elect. Mat. (in press).

15. O. W. Holland, D. K. Thomas, and D. S. Zhou, Appl. Phys. Lett. (submitted).

16. J. A. Brinkman, J. Appl. Phys. 25, 961 (1954).

17. J. A. Brinkman, Amer. J. Phys. 24, 164 (1956).

18. James W. Corbett and James P. Karins, Nucl. Instr. Meth. 182/183, 457 (1981) and references within. 


\section{DISCLAIMER}

This report was prepared as an account of work sponsored by an agency of the United States Government. Neither the United States Government nor any agency thereof, nor any of their employees, makes any warranty, express or implied, or assumes any legal liability or responsibility for the accuracy, completeness, or usefulness of any information, apparatus, product, or process disclosed, or represents that its use would not infringe privately owned rights. Reference herein to any specific commercial product, process, or service by trade name, trademark, manufacturer, or otherwise does not necessarily constitute or imply its endorsement, recommendation, or favoring by the United States Government or any agency thereof. The views and opinions of authors expressed herein do not necessarily state or reflect those of the United States Government or any agency thereof. 